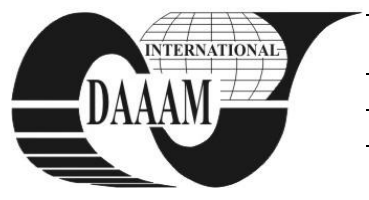

\title{
IMPLEMENTATION OF RIVER INFORMATION SERVICES IN THE REPUBLIC OF CROATIA
}

\author{
KAVRAN, Z[vonko]; JOLIC, N[atalija]; MOSTARAC, K[atarina]; BRNARDIC, M[ato] \& PETROVIC, I[va]
}

\begin{abstract}
Transport of cargo on inland waterways continues to grow, demanding implementation of ICT for increasing transport safety. River information services (RIS) are modern traffic management system, based on ICT, where information are exchanged between vessels and shore. RIS enhance the efficiency of inland navigation using real-time information on every relevant navigation parameter. This paper presents RIS implemented and currently operating in the Republic of Croatia Key words: transport, river information services, system architecture
\end{abstract}

\section{INTRODUCTION}

Development of inland waterway transport is a priority in European Union (NAIADES, 2006) because of its advantages: environmentally - friendly, economical and reliable mode of transport. Future development of inland waterway transport (IWT) sector requires introduction of ICT, intelligent transport systems technologies and solutions. In this way, it can be competitive in global markets, providing accessible, safe, and environmentally - friendly transport networks. RIS enhance the efficiency of inland navigation and facilitate the integration of transport modes through information exchange in the logistics chain management. Transferring freight transport from expensive, congested and often infrastructural inadequate roads and railways to rivers and canals will lead to reduction of fuel consumption, less pollution and will influence economical boost in areas that rely on inland waterway transport.

\section{RELEVANT RIVER INFORMATION SERVICES AND SYSTEM ARCHITECTURE}

\subsection{River information services}

RIS relate to a number of communication devices and protocols that help modernisation of inland waterways. Modern RIS systems consist of number of ICT: hardware, software, communication protocols, human resources and regulation for the purpose of data transmission. Among others (fairway information services, electronic reporting international, etc.), some of the available river information services are listed below.

\subsubsection{Tracking and tracing of vessels}

Tracking and tracing of vessels is constant monitoring of vessels which carry an active Automatic Identification System (AIS) transponder. Through AIS, exchange of relevant information between vessels and vessels and shore (base stations, RIS centers) occur. AIS upon a vessel can automatically identify, locate and track other vessels that are also equipped with an AIS transponder. Information provided by the tracking and tracing of vessel service are: statistical information (name of the vessel, call sign of the vessel, type of vessel, length and width of the vessel), dynamical information (location, speed, route and direction of the vessel, navigation status) and traveling information (length and width of the composition, draught of the vessel, dangerous cargo on board, destination, time of arrival, crew number).

\subsubsection{Notices to Skippers (NtS)}

$\mathrm{NtS}$ are a part of river information services that provide fairway information by broadcasting data messages. These XML (Extended Markup Language) messages are related to identification, fairway, traffic and water level messages and ice warning messages. NtS can be sent by authorities to users (vessels, authorities, RIS centers) and contain information on current fairway situation or warning on potential technical barriers to navigation. $\mathrm{NtS}$ are automatically translated to 21 European languages, which is important for the international exchange and harmonisation of data in different countries.

\subsubsection{Electronic navigation charts (ENC)}

ENC contain relevant cartographic and additional information related to inland navigation. ENC have to be updated, compatible to Inland ECDIS (Electronic Chart Display and Information System) and include minimum data needed for efficient use. Inland ECDIS is a standard for display of electronic navigation charts in inland navigation and can be used in navigation and information mode. Navigation mode uses AIS or radars for obtaining information.

\subsubsection{Calamity Abatement Support (CAS)}

CAS provides assistance with accidents and distress situations, ensuring authorities and rescue forces get useful information on vessels and fairways. For providing efficient CAS, it is assumed that basic river information services are used: tracking and tracing, ENC, NtS, electronic reporting for cargo etc. Skippers, authorities etc. require data related to ship, traffic situation, cargo, crew, hydrometeorology, waterway, and infrastructure. On the other hand, emergency services primarily require specific data on the calamity.

\subsection{System architecture}

Elemental parts of RIS are communication protocols that work as a functional system. Communication can be carried out between RIS centers, between a RIS center and an application, where application can get information from a center, but not the other way around. Internet is used for connecting all of the RIS system stakeholders (RIS centers, vessels, administrators). HTTP client/server enables HTTP communication and provides URL and web interface for system users and system administrators. Backend modules act like national databases where all users and rolls assigned to them are saved. For transmission of data from one point to another, Secure Socket Layer (SSL) is used. This is an open standard that can easily be implemented and provides secure point-to-point connection over IP. SSL verifies identity of the other party and encrypts exchanged data, which secures the connection. For transportation of information HTTP with SOAP protocol is used. Each RIS centre or other user of the system has to support SOAP requests (IRIS Europe Consortium, 2008). Policy Decision Point (PDP) is the unit where the policy decisions are 
made. It gets requests from PEP, XACML Repository (which contains eXtensible Access Control Markup Language rules) and makes decisions. Vessel Index Data Base contains list of ships known to RIS system and has to be harmonised over all RIS centers (national and international). Vessel Location Register contains pointers of tactical traffic information (TTI) which is send by AIS transponder of a certain vessel at some point in time. These pointers have to be saved and not overwritten by new pointers. Public Key Infrastructure is responsible for assignment of all certificates in international exchange of RIS data (client certificates, server certificates, validation and revocation of certificates etc.).

\section{IMPLEMENTATION OF RIS IN THE REPUBLIC OF CROATIA}

RIS are most important segment of modernisation and development of Croatian inland waterways, along with the revitalization of Croatian navigable rivers and ports and their integration to international transport chains. The legal basis for development and implementation of RIS in Croatia are EU Directive 2005/44/EC of the European Parliament and European Council (issued on $7^{\text {th }}$ September 2005) on harmonised river information services (RIS) on inland waterways in the Community (OJ L 255, 30.09.2005), Act on Inland Navigation and Ports (Official Gazette, No. 109/07, 132/07) and Ordinance on River Information Services (CEC, 2007). Implementation of RIS on European inland waterways of Class IV or higher is consisted of three levels of implementation - legal, institutional and technical level (Pacific International Consultants, 2008).

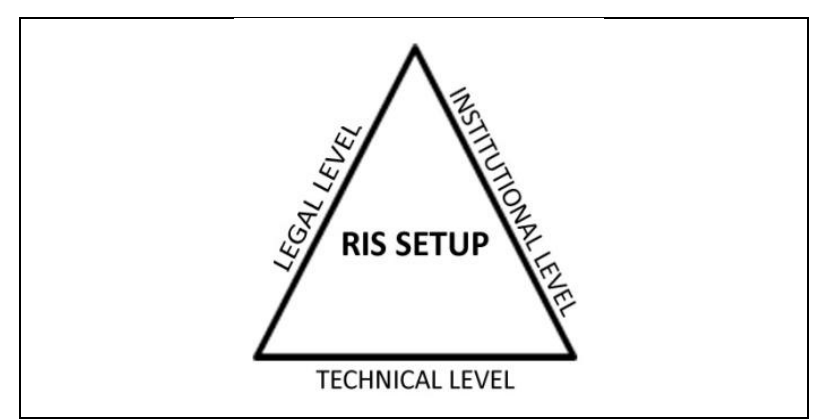

Fig. 1. Levels of implementation of RIS

Approximately $60 \%$ of the major European waterways are provided with ENC, $45 \%$ of the total fleet on the Rhine Danube network is equipped with AIS transponders and ECDIS, NtS are functional in 12 countries, navigation safety is provided throughout VTS/RIS centers at critical stretches along the waterway. For successful RIS implementation in Croatia it is extremely important to apply these levels of implementation and to establish institutions responsible for developing and implementation of appropriate infrastructure, namely hardware and software. Achievement of harmonised and uniform development of RIS infrastructure and services in Croatia, four basic and two additional services need to be established:

1. Tracking and tracing of vessels

2. Notices to Skippers (NtS)

3. Electronic Navigational Charts - ENC/Inland ECDIS

4. VHF voice direct radio link

5. Electronic Reporting International (ERI)

6. Calamity Abatement Service (CAS)

Notices to Skippers are functional at Croatian stretch of the Danube, Drava, Kupa and Sava rivers, and are issued by Harbour Master's Offices Slavonski Brod, Sisak, Osijek and Vukovar. Croatian NtS are in compliance with the international Standard Notices to Skippers that provide standardized data format encoded in a XML file and contain data on identification, fairway and traffic related messages, water level related messages, ice messages and are used for voyage planning or by means of Inland ECDIS for decisions in navigation, short or medium term. Electronic Navigational Charts (ENC) are created for Croatian stretch of the Danube, Drava and Sava rivers, in compliance with international Inland ECDIS Standard versions 1.02 and 2.0. ENC are created for the purpose of decreasing the workload of the skipper during navigation and they do not contain any commercial information. In the scope of Croatian RIS system (CRORIS) five AIS base stations were installed in Osijek, Vukovar, Optaovac, Čvorkovac, Batina and prototype installation has been developed on the Sava river. Existing system completely covers Croatian stretch of Danube and Drava waterway up to Osijek (International Sava River Basin Commission, 2009). The following steps in the further implementation of RIS in Croatia would be to establish a National RIS Center as the owner of the system with the mission of operational management, to develop well structured and organized RIS authorities as well as the environmental aspects in which the service operates. Major research activities would be focused on standardisation and harmonisation scope, because system needs to be complement in entire European waterway network.

\section{CONCLUSION}

Development and implementation of RIS in the Republic of Croatia is important because Croatian waterways need to be fully included in the international waterways and to reach higher international waterway classification levels. Entire Croatian part of the Danube and Drava rivers is fully covered with RIS and further intentions to expand these actions to Sava river have been seriously elaborated. Further measures that would make RIS fully operative in Croatia until 2013 relate to harmonisation with standards and recommendations of EU RIS policies. National RIS Centre should be established and functional, as well as the development of network of well structured and organized RIS authorities and the environment in which the system will operate. Attention should be brought to creation of competitive human resources through education and training of nautical and logistics staff on RIS usage.

\section{REFERENCES}

Grubišić, N (2007): Information System on Inland Waterways RIS, Pomorski zbornik Vol. 40, No. 1, 11/2002, (95 - 111)

CEC (2007): Commission Regulation (EC) No 414/2007 of 13 March 2007 concerning the technical guidelines for the planning, implementation and operational use of River Information Services (RIS), Brussels

European Commission (2006): River information services, as policy implementation flows from research, European Communities, Belgium

International Sava River Basin Commission (2009): Rehabilitation and Development of Transport and Navigation on the Sava River Waterway, Zagreb

Pacific International Consultants (2008): Feasibility Study and Project Documentation for the Rehabilitation and Development of Transport and Navigation on the Sava River Waterway, Zagreb

***IRIS Europe Consortium (2007): IRIS Europe Implementation of River Information Services in Europe: Technical Concept for the Implementation of national and International Exchange of RIS data Available from: http://www.iris-europe.net/download Accessed: 28.08.2011.

***IRIS Europe Consortium (2008): IRIS Europe Implementation of River Information Services in Europe: RIS Data Exchange Process Description Available from: http://www.iris-europe.net/download Accessed: 28.08.2011 